\title{
Visualization of the Trochlear Nerve in the Cistern with Use of High-Resolution Turbo Spin-Echo Multisection Motion-Sensitized Driven Equilibrium
}

\author{
M. Kanoto, Y. Toyoguchi, T. Hosoya, A. Oda, and Y. Sugai
}

\begin{abstract}
BACKGROUND AND PURPOSE: The trochlear nerve is so thin that it is rarely observed with MR imaging. Therefore, we used highresolution MSDE to reliably visualize the cisternal segments of the trochlear nerve.

MATERIALS AND METHODS: Participants were 10 healthy young adults (mean age, 24 years), and 20 trochlear nerves were examined. HR-MRC, BS-MRC, and HR-MSDE were performed. A neuroradiologist judged the visibility of the trochlear nerves as 1 of 4 grades ("Excellent," "Good," "Fair," and "Not") in each MR imaging sequence. The findings were then statistically analyzed with the $\chi^{2}$ test.

RESULTS: Of all 20 trochlear nerves, 6 with HR-MRC, 13 with BS-MRC, and 18 with HR-MSDE were judged as "Excellent." CSF flow-related artifacts and vessels in the cistern and cerebellar tentorium in HR-MRC tended to prevent the neuroradiologists from identifying the trochlear nerve. Vessels in the cistern and cerebellar tentorium in BS-MRC also tended to prevent the neuroradiologists from identifying the trochlear nerve. Compared with other sequences, HR-MSDE visualized the trochlear nerve more often. The $\chi^{2}$ test revealed statistically significant differences among the $3 \mathrm{MR}$ imaging sequences $(P<.01)$. The scan time of HR-MSDE was approximately $1.5-2.2$ times longer than that of the other sequences.
\end{abstract}

CONCLUSIONS: HR-MSDE is able to clearly visualize the trochlear nerve and has the same or better ability to delineate the trochlear nerve compared with other MR imaging sequences, though its long scan time does not yet yield practical use.

ABBREVIATIONS: $\quad B S=$ balanced sequence; $H R=$ high-resolution; $M R C=M R$ cisternography; $M S D E=$ motion-sensitized driven equilibrium; $S A R=$ specific absorption rate

T rochlear nerve palsy is clinically characterized by vertical diplopia (incomitant hypertropia that increases on tilting of the head toward the paralyzed site [Bielschowsky test]), excyclotropia, and head tilt. ${ }^{1,2}$ The trochlear nerve is easily injured by surgery, head trauma, brain herniation, and other insults. ${ }^{3}$ Imaging assessment of the trochlear nerve is more difficult than other cranial nerves, as the fourth cranial nerve is much thinner than the other cranial nerves and has a complex course in the basal cisterns.

Received July 31, 2012; accepted after revision September 28.

From the Department of Diagnostic Radiology, Yamagata University Faculty of Medicine, Yamagata, Japan.

This work was supported by a grant-in-aid from the Global COE (Centers of Excellence) Program of the Japan Society for the Promotion of Science.

Paper previously presented at: Annual Meeting of the Japanese Society of Neuroradiology, Tokyo, Japan, February 2011, and Annual Meeting of the European Society of Neuroradiology, Antwerp, Belgium, September 2011.

Please address correspondence to Masafumi Kanoto, MD, PhD, Department of Diagnostic Radiology, Yamagata University Faculty of Medicine, lida-Nishi 2-2-2, Yamagata, Japan 990-9585; e-mail: mkanoto@med.id.yamagata-u.ac.jp

- Indicates open access to non-subscribers at www.ajnr.org

http://dx.doi.org/10.3174/ajnr.A3403

The trochlear nerve is the only cranial nerve that originates from the mesencephalic dorsum and runs along the cerebellar tentorium as it courses to the cavernous sinus. ${ }^{4-7}$ Arteries and veins surround the trochlear nerve in a complicated manner. Given the small size of the nerve and complex cisternal anatomy, it has been difficult to reliably identify the trochlear nerve by MR imaging and other imaging modalities.

TSE-MSDE is a new MR imaging technique similar to diffusionweighted imaging that has been applied to the evaluation of atherosclerotic plaque in the coronary and carotid arteries, as well as brain metastases. ${ }^{8,9}$ TSE-MSDE has also been applied for assessment of the existence of brain metastasis with contrast media. ${ }^{10}$ This method takes advantage of a preparative sequence, a diffusion pulse with a very low b-value consisting of 3 nonselective radio-frequency pulses with flip angles of $90-180-90^{\circ}$, and symmetric gradients around the $180^{\circ}$ pulse. It causes phase dispersion of blood and CSF spins by using a magnetic field gradient and suppresses any flow signals. ${ }^{11}$ TSEMSDE is able to clearly show only cranial nerves in the cistern with no flow signal. ${ }^{12}$ We hypothesized that TSE-MSDE may be able to effectively visualize the trochlear nerve because it can suppress any signals in the cistern. The purpose of our study was to assess visualization of the trochlear nerve in the cistern by using TSE-MSDE. 


\section{MATERIALS AND METHODS}

This protocol was approved by our ethics committee.

\section{Participants}

Participants were 10 healthy young adults (6 men and 4 women; mean age, 24 years; age range, 21-28 years), and 20 trochlear nerves were examined. We obtained informed consent from these volunteers before performing MR imaging. We verbally confirmed that they had never undergone surgery or had any significant or relevant prior medical problems.

\section{MR Imaging}

We performed $3 \mathrm{MR}$ imaging sequences for each participant by using a 3T MR imaging unit (Intera Achieva; Philips Healthcare, Best, the Netherlands). We modified the conventional MRC and TSE-MSDE to visualize the trochlear nerve and created HR-MRC and HR-MSDE protocols. In addition to these MR imaging sequences, we also performed BS-MRC, according to a previous study. ${ }^{13}$ The imaging parameters were as follows. For HR-MRC, TR was $3000 \mathrm{~ms}$; TE, $287 \mathrm{~ms}$; acquisition voxel size, $0.30 \times 0.33 \times$ $0.60 \mathrm{~mm}$; reconstruction voxel size, $0.21 \times 0.21 \times 0.30 \mathrm{~mm}$; NEX, 1; FOV, $150 \mathrm{~mm}$; flip angle, $90^{\circ}$; number of sections, 80 ; section thickness, $0.30 \mathrm{~mm}$; coil, sensitivity encoding 8 -channel head coil; scan time, 18 minutes 3 seconds; and SAR/head, $0.9 \mathrm{~W} / \mathrm{kg}$. For the balanced sequence, TR was $9.0 \mathrm{~ms}$; TE, $3.5 \mathrm{~ms}$; acquisition voxel size, $0.29 \times 0.30 \times 0.50 \mathrm{~mm}$; reconstruction voxel size, $0.29 \times$ $0.29 \times 0.25 \mathrm{~mm}$; NEX, 1 ; FOV , $150 \mathrm{~mm}$; flip angle, $60^{\circ}$; number of sections, 60 ; section thickness, $0.25 \mathrm{~mm}$; coil, sensitivity encoding 8-channel head coil; scan time, 11 minutes 59 seconds; and SAR/ head, $3.2 \mathrm{~W} / \mathrm{kg}$. For HR-MSDE, TR was $24 \mathrm{~ms}$; TE, $4 \mathrm{~ms}$; acquisition voxel size, $0.39 \times 0.38 \times 0.50 \mathrm{~mm}$; reconstruction voxel size, $0.31 \times 0.31 \times 0.25 \mathrm{~mm}$; NEX, 1; FOV, $200 \mathrm{~mm}$; flip angle, $20^{\circ}$; b-value, 10; number of sections, 120 ; section thickness, 0.25 $\mathrm{mm}$; coil, sensitivity encoding 8 -channel head coil; scan time, 26 minutes 37 seconds; and SAR/head, $0.6 \mathrm{~W} / \mathrm{kg}$. These sequences were prescribed in the axial plane, and the scan range was set so that the mesencephalon and cavernous sinuses were included.

\section{Image Analysis}

Two experienced neuroradiologists (A: Y.T. and B: M.K., with 7 and 10 years of experience, respectively) evaluated the MR images and separately determined the visibility of each trochlear nerve.

First, neuroradiologist A evaluated whether the trochlear nerve was identified in full length in the cistern (from the quadrigeminal cistern to the entrance of the cavernous sinus). We classified the visibility of trochlear nerve into 4 grades: "Excellent," "Good," "Fair," and "Not." A grade of "Excellent" was given if more than $90 \%$ of full length was identified; "Good," if 50\%-90\% of full length was identified; "Fair," for $10 \%-50 \%$ of full length; and "Not," for less than $10 \%$ of full length.

Next, we divided the trochlear nerve into 2 segments-the perimesencephalic segment and the tentorial segment-by using a line that links the mesencephalic tegmentum-cerebral peduncle junction to the cerebellar anterior border in the same section (Fig 1). ${ }^{6}$ The perimesencephalic segment was defined as the range from the root exit point of the trochlear nerve in the dorsal mesencephalon to the ambient cistern. The tentorial segment was defined as the range from the portion along the cerebellar tentorium to the cavernous sinus. If the nonbranching curvilinear structure was evident in the ambient cistern and along the cerebellar tentorium, it was identified as the trochlear nerve. The visibility of the trochlear nerve in each segment was evaluated by 2

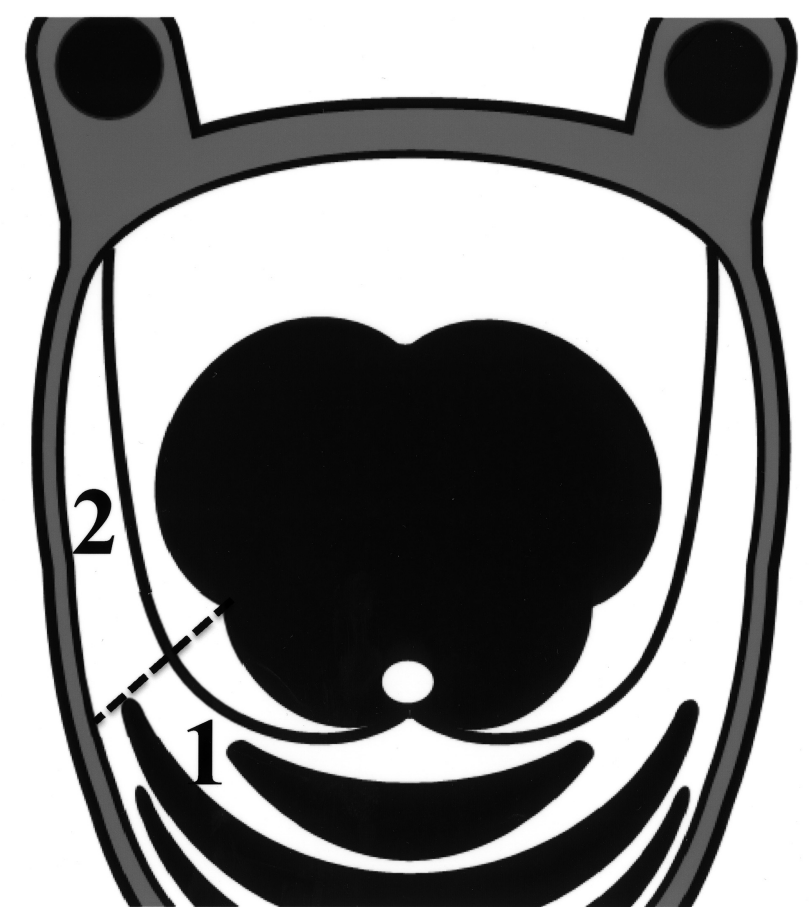

FIG 1. Segmentation of the trochlear nerve. The trochlear nerve in the cistern was divided into 2 segments: the perimesencephalic segment and the tentorial segment, by use of the line that links the mesencephalic tegmentum-cerebral peduncle junction to the cerebellar anterior border in the same section. 1. Perimesencephalic segment. 2. Tentorial segment.

\section{Table 1: Visibility of 20 trochlear nerves in full length}

\begin{tabular}{lccc}
\hline Visibility & HR-MRC & BS-MRC & HR-MSDE \\
\hline Excellent & 6 & 13 & 18 \\
Good & 5 & 2 & 1 \\
Fair & 9 & 4 & 1 \\
Not & 0 & 1 & 0 \\
\hline
\end{tabular}

neuroradiologists. We observed all images by using a DICOM viewer (Philips Healthcare) and by paging all sections. The viewers could scroll through the sections and change appropriate window/level magnification. The visibility of each sequence was statistically analyzed by use of the $\chi^{2}$ test, and we calculated the $\kappa$ coefficient between the 2 neuroradiologists for each MR imaging sequence and segment to evaluate the concordance of the findings between them (SPSS, version 16.0; SPSS, Chicago, Illinois).

\section{RESULTS}

\section{Visibility of the Trochlear Nerve in Full Length}

The visibility of the trochlear nerve in full length is summarized in Table 1. Of all 20 trochlear nerves, 6 with HR-MRC, 13 with BS-MRC, and 18 with HR-MSDE were judged as "Excellent" by a neuroradiologist. With HR-MRC, CSF flow-related artifacts and vessels in the cistern and/or cerebellar tentorium tended to prevent the neuroradiologists from identifying the trochlear nerve (Fig 2A). With BS-MRC, vessels in the cistern and the cerebellar tentorium also tended to prevent the neuroradiologists from identifying the trochlear nerve (Fig $2 B$ ). HR-MSDE more often visualized the trochlear nerve compared with the other sequences (Fig 2C). The $\chi^{2}$ test revealed statistically significant differences among these $3 \mathrm{MR}$ imaging sequences $(P<.01)$. 

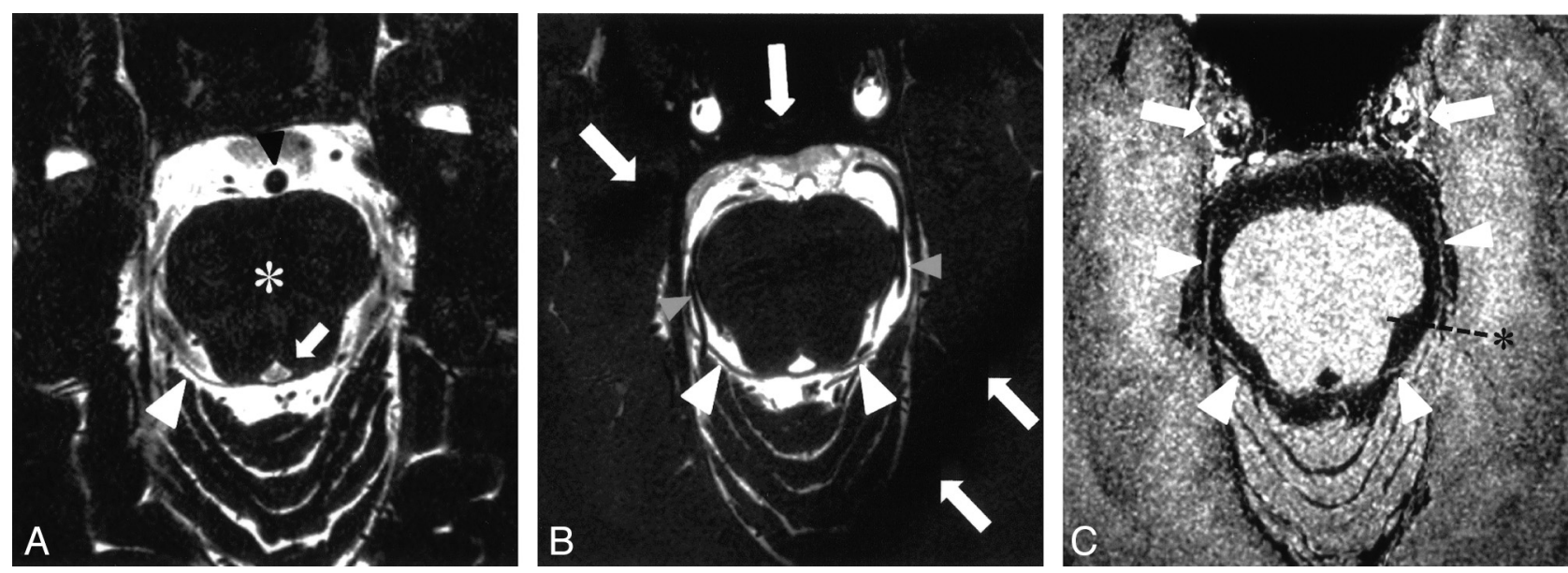

FIG 2. MR images of each sequence. A, HR-MRC: Perimesencephalic segment of the right trochlear nerve is observed (white arrowhead). Marked CSF flow-related artifacts in the cistern are apparent. The basilar artery (black arrowhead), midbrain (*), and cerebral aqueduct (white arrow) are noted. B, BS-MRC: Bilateral trochlear nerves are apparent in the cistern (arrowheads). The bilateral posterior cerebral artery is observed around the trochlear nerve (gray arrowheads). Band artifacts are also evident (white arrows). C, HR-MSDE: Trochlear nerves are visualized as curvilinear structures in full length (arrowheads). The line for segmentation (*) and the carotid artery (white arrows) are observed.

Table 2: Visibility of 20 trochlear nerves in each segment between 2 neuroradiologists

\begin{tabular}{|c|c|c|c|c|c|c|}
\hline \multirow{2}{*}{$\begin{array}{l}\text { Segment of the } \\
\text { Trochlear Nerve }\end{array}$} & \multicolumn{2}{|c|}{ HR-MRC } & \multicolumn{2}{|c|}{ BS-MRC } & \multicolumn{2}{|c|}{ HR-MSDE } \\
\hline & A & B & A & B & A & B \\
\hline Perimesencephalic & 19 & 14 & 19 & 18 & 20 & 20 \\
\hline Tentorial* & 7 & 2 & 13 & 14 & 19 & 18 \\
\hline
\end{tabular}

A indicates neuroradiologist $A ; B$, neuroradiologist $B$

* Significant differences among 3 MRI sequences by the $\chi^{2}$ test $(P<.01)$.

\section{Visibility of the Trochlear Nerve in the Perimesencephalic Segment}

The visibility of the trochlear nerve in the perimesencephalic segment is summarized in Table 2. HR-MRC, BS-MRC, and HRMSDE had approximately equal trochlear nerve visualization performance. For neuroradiologist B, the $\chi^{2}$ test revealed statistically significant differences among these $3 \mathrm{MR}$ imaging sequences $(P=.02)$.

\section{Visibility of the Trochlear Nerve in the Tentorial Segment}

The visibility of the trochlear nerve in the tentorial segment is summarized in Table 2. As with the evaluation in full length, CSF flow-related artifacts, vessels in the cistern, and cerebellar tentorium in HR-MRC tended to prevent both neuroradiologists from identifying the trochlear nerve (Fig 2A). Vessels in the cistern and cerebellar tentorium in BS-MRC also tended to prevent both neuroradiologists from identifying the trochlear nerve (Fig 2B). HRMSDE more often visualized the trochlear nerve compared with the other sequences (Fig 2C). For both neuroradiologists A and B, the $\chi^{2}$ test revealed statistically significant differences among these $3 \mathrm{MR}$ imaging sequences $(P<.01)$.

\section{Visibility Concordance between 2 Neuroradiologists}

The concordance of visibility of the trochlear nerve between the 2 neuroradiologists is summarized in Table 3. There were high concordances in BS-MRC and HR-MSDE $(\kappa=0.643-0.659)$. In the tentorial segment of HR-MSDE, the $\kappa$ coefficient could not be calculated because both neuroradiologists evaluated the trochlear nerve as "visible" in all cases. In HR-MRC, there was a low concordance between the 2 radiologists.
Table 3: Concordance in each MR imaging sequence between 2 neuroradiologists

\begin{tabular}{lccc}
\hline \multirow{2}{*}{$\begin{array}{l}\text { Segment of the } \\
\text { Trochlear Nerve }\end{array}$} & HR-MRC & BS-MRC & HR-MSDE \\
\cline { 2 - 4 } Perimesencephalic & 0.286 & 0.643 & $-{ }^{*}$ \\
Tentorial & 0.219 & 0.659 & 0.643 \\
\hline
\end{tabular}

* The $\kappa$ coefficient could not be calculated because both neuroradiologists evaluated the trochlear nerve as "visible" in all cases.

\section{DISCUSSION}

Our study demonstrates that we can almost always observe the cisternal portion of the trochlear nerve by using HR-MSDE. The trochlear nerve, the diameter of which ranges from approximately 0.3 to $0.9 \mathrm{~mm}$, is the thinnest of all cranial nerves. ${ }^{13}$ Moreover, it has a complex $3 \mathrm{D}$ course around the midbrain, and many arteries and veins, as well as the cerebellar tentorium, are in close proximity to the nerve. ${ }^{4-7}$ Thus, it has been difficult to identify the trochlear nerve by using a variety of even HR MR imaging sequences. We demonstrated that HR-MSDE was significantly superior to both HR-MRC and BS-MRC in visualizing the trochlear nerve, with high concordance between 2 neuroradiologists.

The perimesencephalic segment of the trochlear nerve was generally well identified on all MR images used. This is probably because of the course of the nerve around the midbrain. The trochlear nerve originates from the root exit zone in the dorsal pontomesencephalic junction and runs a curved course that differs from the course of the vessels in the perimesencephalic cistern. In contrast, the tentorial segment of the trochlear nerve tended to be obscured on the MRC images, as it runs along the tentorial edge and is parallel to the vessels in its tentorial segment. Therefore, the trochlear nerve may be difficult to differentiate from the tentorial edge and/or vessels on MRC images because both nerves and vessels are rendered with low intensity.

TSE-MSDE provides 3D T1 contrast images that can nearly entirely suppress flow signals by using a preparation pulse. Previous reports ${ }^{8-12}$ have discussed TSE-MSDE regarding the atherosclerotic change in vessels, the existence of brain metastasis, and focal deformation of nerves by vessels in neurovascular compression syndrome. In our study, we obtained HR images with TSEMSDE (ie, HR-MSDE) to visualize the trochlear nerve. The flow signals from vessels and CSF were well suppressed, and only the 
cranial nerves were clearly visualized in the cistern. In addition, the signal of the cerebellar tentorium was also suppressed because its low T1 contrast was the same as that of CSF. Thus, the trochlear nerve was well visualized not only in its perimesencephalic segment but also in its tentorial segment. Because the oculomotor nerve and the trigeminal nerve are much thicker than the trochlear nerve, these cranial nerves were clearly visualized in the same scan range. As MR imaging contrast media can be used in conjunction with HR-MSDE, we may in the future be able to visualize actual lesions causing trochlear nerve paralysis by using this method and applying it to clinical cases.

MRC is generally an adequate method to delineate cranial nerves and related vessels. ${ }^{14}$ The trochlear nerve is so thin, however, that much thinner sections are required to visualize it than are routinely acquired. If very thin sections are acquired, then prominent flow-related artifacts will occur as a result of CSF, and this mechanism creates a significant problem in the observation of the nerves and vessels. Indeed, the trochlear nerve was poorly identified on HR-MRC images, especially in its tentorial segment.

In general, 3D constructive interference in steady state and fast imaging employing steady-state acquisition visualize the cranial nerves well, such as the oculomotor, trigeminal, abducens, facial, auditory, and lower cranial nerves, but the trochlear nerve is an exception. ${ }^{15-21}$ Cranial nerves that penetrate the cavernous sinus, such as the oculomotor, trochlear, trigeminal, and abducens nerve, can be visualized by $3 \mathrm{D}$ constructive interference in steady state. ${ }^{21}$ However, it has been difficult to visualize the trochlear nerve in its cisternal segment. Choi et $\mathrm{al}^{13}$ reported that the trochlear nerve can be clearly visualized with HR 3D balanced turbo-field echo. We applied this method in our MR imaging unit and obtained BS-MRC. It is easier to identify the trochlear nerve with BS-MRC compared with HR-MRC because there is decreased influence of flow-related artifacts from CSF. ${ }^{22}$ However, band artifacts often occur in the peripheral area of the cistern on BS-MRC images, and there is a scan range limit. Thus, we can only observe the central area in the area of interest, and the observable area is narrow.

The major limitation of our study was the long scan time required. It took approximately 30 minutes for HR-MSDE; therefore, the scan time is so long that this sequence is not yet of practical use. In our study, we acquired 120 sections in HR-MSDE to visualize a thin nerve, the trochlear nerve, with HR and high signal-to-noise ratio. If the number of sections is reduced, then signal-to-noise ratio decreases, and it may become impossible to identify the trochlear nerve. A much shorter scan time for this sequence for practical use should be achievable through the improvement of sequence parameters or the use of a 7T MR imaging unit in the future. Decreased scan time would also allow application of this method in pediatric cases (eg, congenital palsy of the superior oblique muscle). In our study, however, the scan time was not able to be reduced anymore; therefore, it is impossible to apply this method to pediatric cases at present. We also acknowledge that our BS-MRC was a slightly different sequence from the originally reported one, as we had to adjust parameters for use on a different MR imaging machine.

\section{CONCLUSIONS}

HR-MSDE is clearly able to visualize the trochlear nerve and, in general, has a better ability to delineate the trochlear nerve compared with other MR imaging sequences. However, the scan time is so long that it is not yet feasible for routine clinical use.
Disclosures: Masafumi Kanoto-RELATED: Grant: Grant-in-aid from the Global COE (Centers of Excellence) Program of the Japan Society for the Promotion of Science.* ( ${ }^{*}$ Money paid to institution.)

\section{REFERENCES}

1. Bazan R, Braga GP, Gomes DL, et al. Trochlear nerve palsy associated with Claude Bernard-Horner syndrome after brainstem stroke. Case Rep Neurol 2011;3:248-51

2. Bababeygy SR, Quiros PA. Isolated trochlear palsy secondary to Lyme neuroborreliosis. Int Ophthalmol 2011;31:493-95

3. van Overbeeke, JJ, Cruysberg JR, Menovsky T. Intracranial repair of a divided trochlear nerve. Case report. J Neurosurg 1998;88:336-39

4. Bisaria KK. Cavernous portion of the trochlear nerve with special reference to its site of entrance. J Anat 1988;159:29-35

5. Tubbs RS, Oakes WJ. Relationships of the cisternal segment of the trochlear nerve. J Neurosurg 1998;89:1015-19

6. Ammirati M, Musumeci A, Bernardo A, et al. The microsurgical anatomy of the cisternal segment of the trochlear nerve, as seen through different neurosurgical operative windows. Acta Neurochir (Wien) 2002;144:1323-27

7. Yousry I, Moriggl B, Dieterich M, et al. MR anatomy of the proximal cisternal segment of the trochlear nerve: neurovascular relationships and landmarks. Radiology 2002;223:31-38

8. Wang J, Yarnykh VL, Yuan C. Enhanced image quality in blackblood MRI using the improved motion-sensitized driven-equilibrium (iMSDE) sequence. J Magn Reson Imaging 2010;31:1256-63

9. Nguyen TD, de Rochefort L, Spincemaille P, et al. Effective motionsensitizing magnetization preparation for black blood magnetic resonance imaging of the heart. J Magn Reson Imaging 2008;28:1092-100

10. Nagao E, Yoshiura T, Hiwatashi A, at al. 3D turbo spin-echo sequence with motion-sensitized driven-equilibrium preparation for detection of brain metastases on 3T MR imaging. AJNR Am J Neuroradiol 2011;32:664-70

11. Wang J, Yarnykh VL, Hatsukami T, et al. Improved suppression of plaque-mimicking artifacts in black-blood carotid atherosclerosis imaging using a multislice motion-sensitized driven-equilibrium (MSDE) turbo spin-echo (TSE) sequence. Magn Reson Med 2007;58: 973-81

12. Kanoto M, Hosoya T, Oda A, et al. Focal deformity of the cranial nerves observed on multislice motion-sensitized driven equilibrium (MSDE) in patients with neurovascular compression. J Comput Assist Tomogr 2012;36:121-24

13. Choi BS, Kim JH, Jung C, et al. High-resolution 3D MR imaging of the trochlear nerve. AJNR Am J Neuroradiol 2010;31:1076-79

14. Mamata Y, Muro I, Matsumae M, et al. Magnetic resonance cisternography for visualization of intracisternal fine structures. $\mathrm{J} \mathrm{Neu}$ rosurg 1998;88:670-78

15. Cheng YS, Zhou ZR, Peng WJ, et al. Three-dimensional-fast imaging employing steady-state acquisition and T2-weighted fast spin-echo magnetic resonance sequences on visualization of cranial nerves III - XII. Chin Med J (Engl) 2008;121:276-79

16. Fischbach F, Müller M, Bruhn H. High-resolution depiction of the cranial nerves in the posterior fossa (N III-N XII) with 2D fast spin echo and 3D gradient echo sequences at 3.0 T. Clin Imaging 2009;33: $169-74$

17. Zhang Z, Meng Q, Chen $Y$, et al. 3-T imaging of the cranial nerves using three-dimensional reversed FISP with diffusion-weighted MR sequence. J Magn Reson Imaging 2008;27:454-58

18. Yousry I, Camelio S, Schmid UD, et al. Visualization of cranial nerves I-XII: value of 3D CISS and T2-weighted FSE sequences. Eur Radiol 2000;10:1061-67

19. Yousry I, Moriggl B, Schmid UD, et al. Trigeminal ganglion and its divisions: detailed anatomic MR imaging with contrast-enhanced $3 \mathrm{D}$ constructive interference in the steady state sequences. AJNR Am J Neuroradiol 2005;26:1128-35

20. Amemiya S, Aoki S, Ohtomo K. Cranial nerve assessment in cavernous sinus tumors with contrast-enhanced 3D fast-imaging employing steady-state acquisition MR imaging. Neuroradiology 2009;51: 467-70

21. Yagi A, Sato N, Taketomi A, et al. Normal cranial nerves in the cavernous sinuses: contrast-enhanced three-dimensional constructive interference in the steady state MR imaging. AJNR Am J Neuroradiol 2005;26:946-50

22. Scheffler K, Lehnhardt S. Principles and applications of balanced SSFP techniques. Eur Radiol 2003;13:2409-18 